\title{
Reaction Monitoring Using Mid-Infrared Laser-Based Vibrational Circular Dichroism
}

Anja Rüther, ${ }^{1}$ Marcel Pfeifer, ${ }^{2}$ Víctor A. Lórenz-Fonfría, ${ }^{3}$ and Steffen Lüdeke ${ }^{1 *}$

${ }^{1}$ Institute of Pharmaceutical Sciences, Albert-Ludwigs-Universität Freiburg, Albertstr. 25, 79104 Freiburg, Germany

${ }^{2}$ Fraunhofer Institute for Physical Measurement Techniques, Heidenhofstr. 8, 79110 Freiburg

${ }^{3}$ Freie Universität Berlin, Experimental Molecular Biophysics, Arnimallee 14, 14195 Berlin, Germany

Shortened title:

Reaction monitoring using laser-based VCD

Keywords:

Vibrational circular dichroism; hydrolytic reaction; flow-through setup; quantum cascade laser; time-resolved spectroscopy; global exponential fitting; matrix least squares fitting

*Correspondence to:

Dr. Steffen Lüdeke

Institute of Pharmaceutical Sciences

Albertstr. 25, D-79104 Freiburg, Gemany

E-mail: steffen.luedeke@pharmazie.uni-freiburg.de

Tel.: +49 (0)761 203-6353

Fax: $+49(0) 761203-6351$

Additional Supporting Information may be found in the online version of this article.

Contract grant sponsor: Baden-Württemberg Stiftung (Eliteprogramme for Postdocs)

Contract grant number: 1.16101 .09 


\section{Abstract}

Changes in vibrational circular dichroism (VCD) were recorded on-line during a chemical reaction. The chiral complex nickel-(-)-sparteine chloride was hydrolyzed to free (-)sparteine base in a biphasic system of sodium hydroxide solution and chloroform $\left(\mathrm{CHCl}_{3}\right)$. IR and VCD spectra were iteratively recorded after pumping a sample from the $\mathrm{CHCl}_{3}$ phase through a lab-built VCD spectrometer equipped with a tunable mid-infrared quantum cascade laser light source, which allows for VCD measurements even in the presence of strongly absorbing backgrounds. Time-dependent VCD spectra were analyzed by singular value decomposition and global exponential fitting. Spectral features corresponding to the complex and free (-)-sparteine could be clearly identified in the fitted amplitude spectrum, which was associated to an exponential decay with an apparent time constant of $127 \mathrm{~min}\left(t_{1 / 2}=88 \mathrm{~min}\right)$.

\section{Introduction}

Due to their high sensitivity towards changes in a molecule's three-dimensional geometry, chiroptical spectroscopy methods have a high potential for being used for studying timedependent dynamic processes. Introduced for studies on nano-second time-scale photolysis of (carbonmonoxy)myoglobin, ${ }^{1}$ time-resolved electronic circular dichroism (ECD) has been applied for studies involving both small ${ }^{2}$ and biological molecules. ${ }^{3}$ Vibrational circular dichroism (VCD), the difference in absorbance of left minus right circularly polarized infrared (IR) light, combines the virtues of both the high content of information of vibrational spectra and the sensitivity to a molecule's three dimensional properties from CD. It is, in combination with quantum chemical calculations, widely used for the determination of the absolute configuration. ${ }^{4} \mathrm{VCD}$ is also very sensitive towards conformational equilibria, ${ }^{5}$ and secondary structure, $^{6}$ thereby complementing ECD. These properties render the application for monitoring of time-dependent processes highly desirable. However, VCD is generally restricted to solutions in IR-transmissible solvents such as $\mathrm{CDCl}_{3}, \mathrm{CCl}_{4}$, and optimal concentrations and path lengths - conditions that are not compatible with most chemical reactions. Nevertheless, it could be shown that use of a quantum cascade laser (QCL) as a light source, with its high output power, permits the measurement of VCD spectra of highly absorbing samples, too. ${ }^{7}$

A simple example for a time-dependent change of optical activity is a racemization process. The potential of VCD for monitoring the time-dependent change of enantiomeric excess has previously been demonstrated through different proof-of-concept experiments. ${ }^{8,9}$ According to Beer's law, vibrational spectra of chemical compositions represent a linear combination of 
spectra of the involved compounds, also allowing for quantitative analysis. ${ }^{10}$ In time-resolved experiments, these spectra correspond to species that are present at a certain point in time. Therefore, a model to describe the VCD monitoring of a racemization would involve the spectra of two extrema: the full amplitude of the pure enantiomer at the beginning, and a zero line after full racemization. Chemical reactions that involve the conversion of one compound into another, however, might require more complicated models. Provided that a reaction can be approximated as first order, time-resolved spectra can be modeled as the sum of contributions from one or more exponential decays with the use of global fitting procedures. This concept has for example been used to deconvolute time-resolved spectral data of lightinduced reactions in photo-sensitive proteins. ${ }^{11}$

In order to demonstrate the applicability of QCL-based VCD as a detection device in a flowthrough setup for monitoring a chemical conversion, we recorded VCD spectra of the hydrolysis of nickel-(-)-sparteine chloride (1) to sparteine (2) at the interface between the organic solvent $\left(\mathrm{CHCl}_{3}\right)$ and an aqueous sodium hydroxide solution over time (Scheme 1).

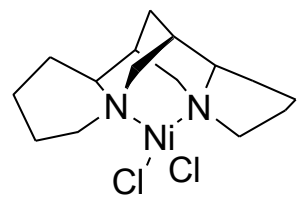

1

$-2 \mathrm{Cl}^{-} \downarrow \uparrow+2 \mathrm{Cl}^{-}$

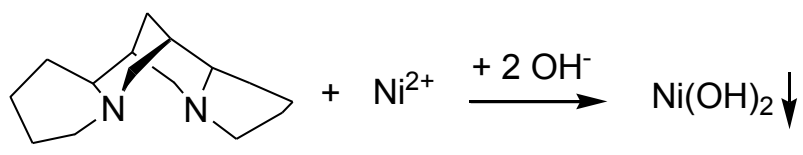

2

Scheme 1: Reaction sequence of the basic hydrolysis of nickel-(-)-sparteine chloride (1) to sparteine (2), involving dissociation of the complex and precipitation of $\mathrm{Ni}(\mathrm{OH})_{2}$.

The high spin complex 1 exhibits strong VCD due to low-lying $\mathrm{d}-\mathrm{d}$ transitions leading to enhanced rotational strength according to vibronic coupling theory. ${ }^{12-14}$ Upon hydrolysis of 1 to 2 these $d-d$ transitions are removed, which results into characteristic changes in the spectral range $\left(1322.5-1226.5 \mathrm{~cm}^{-1}\right)$ observed with the QCL-VCD setup. 
The reaction consists of a dissociation and a precipitation step (Scheme 1) and depends on different reactants, products and intermediates. Furthermore, one should be aware of potential contributions to the kinetics of the reaction from diffusion of reactants to the chloroform/water interface. Nevertheless, the kinetic description of the observable decrease of the concentration of $\mathbf{1}$ in the chloroform phase may be simplified after certain considerations are taken into account. The very low solubility of $\mathrm{Ni}(\mathrm{OH})_{2}$ in water $\left(K=10^{-16.1} \mathrm{~mol}^{3} \mathrm{~L}^{-3}\right)^{15}$ suggests fast precipitation at the interface, which should not be rate limiting. Because $\mathrm{Ni}^{2+}$ is rapidly removed from the solution, the back reaction (formation of 1) should be negligible, which renders the dissociation step a pseudo-first order reaction. The reaction predominantly happens at the interface. Therefore, the reaction rate may be limited by diffusion, too. This influence, however can be minimized by stirring. Still, the area of the interface may contribute as a constant parameter to the observed reaction rate of the overall pseudo-first order reaction determined by the dissociation step. In conclusion, we hypothesize that the decrease of $\mathbf{1}$, observable as time-dependent spectral changes, can be described by a single exponential decay:

$$
A(\widetilde{v}, t)=a_{\infty}(\widetilde{v})+a(\widetilde{v}) e^{-\frac{t}{\tau}}
$$

$A$ is a time-dependent spectrum measured in the course of the reaction. The amplitude spectrum $a_{\infty}$ represents a time-independent component, which corresponds to the VCD spectrum of 2. The decay associated amplitude $a$ corresponds to the VCD spectrum of $\mathbf{1}$ minus the VCD spectrum of 2.

We subsequently recorded VCD spectra of the reaction solution with changing content of 1 and 2 over time. Using global exponential fitting to the time-dependent VCD data set, we obtained a time constant for the hydrolysis of $\mathbf{1}$ and an amplitude spectrum that can be attributed to spectral features that correspond to $\mathbf{1}$ and $\mathbf{2}$.

\section{Materials and Methods}

\section{IR- and VCD reaction monitoring}

For time-dependent IR and VCD measurements we used a setup similar to the one described previously. ${ }^{7}$ Circularly polarized light was generated from linearly polarized radiation of the external cavity QCL (Daylight Solutions, San Diego, CA, USA) by a $70 \mathrm{kHz}$ photoelastic modulator (PEM 80, Hinds, Hillsboro, OR) with anti-reflective coating to reduce artifacts from laser interference. Intensities were recorded using a liquid $\mathrm{N}_{2}$ cooled detector (L-8575 
HCT-70, InfraRed Associates Inc., Stuart, FL). Modulation of the light-beam by a mechanical chopper allowed for AC coupling of the detector. Intensities corresponding to the IR and the VCD signal were obtained from demodulation by two lock-in amplifiers (SRS830, Stanford Research Systems, Sunnyvale, CA, USA) referenced to chopper and PEM frequencies, respectively.

The nickel-(-)-sparteine chloride complex 1 was synthesized according to literature. ${ }^{16}(-)$ Sparteine (2) was obtained after dissolving (-)-sparteine sulphate (Fluka, Buchs, Switzerland) in a $100 \mathrm{mM}$ sodium hydroxide solution and extraction with tert-butyl methyl ether. For hydrolysis of 1 a $200 \mathrm{mM}$ solution in $\mathrm{CHCl}_{3}(326 \mathrm{mg}$ in $3 \mathrm{~mL}$ ) was overlayed with $5 \mathrm{~mL}$ of a $200 \mathrm{mM}$ aqueous sodium hydroxide solution in a test tube. Though the organic phase was stirred vigorously, care was taken not to perturb the phase boundary, where the hydrolysis took place. In the course of the reaction, $\mathrm{Ni}(\mathrm{OH})_{2}$ precipitated as a lime-green solid, whereas free base 2 accumulated in the $\mathrm{CHCl}_{3}$ phase.

Independent static spectra of $\mathbf{1}$ were recorded before the reaction was started, averaged and used as a background for time-dependent spectra. QCL-IR and QCL-VCD measurements were undertaken every 20 minutes by aspirating a sample from the organic layer and pumping it through a $200 \mu \mathrm{m} \mathrm{CaF} 2$ sample cell, which rested in the light-beam throughout the whole experiment and was connected to the reaction vessel by Teflon tubing. Using a three-way valve (NResearch Inc., West Caldwell, NJ, USA) virtually full exchange of sample between two measurements was established. Referencing to the time when the sodium hydroxide layer was added, we recorded 19 spectra after reaction times of 5, 27, 48, 71, 96, 117, 141, 164, 186, $210,232,253,276,298,320,342,364,386$, and $408 \mathrm{~min}$. The QCL scanned with a nominal step size of $0.5 \mathrm{~cm}^{-1}$ between 1320 and $1230 \mathrm{~cm}^{-1}$ except for spectral regions from 1279 to $1272.5,1255$ to 1248,1240 to $1235.5 \mathrm{~cm}^{-1}$, where a nominal step size of $0.25 \mathrm{~cm}^{-1}$ was used. The different step sizes were necessary in order to avoid gaps between two sampling points, which arise after recalibration of nominal to actual frequencies (actual spectral range: 1322.5 to $1226.5 \mathrm{~cm}^{-1}$ ) from inaccuracies in the frequency calibration of the laser. Each frequency data point is an average of 50 lock-in readings (time constant $300 \mathrm{~ms}$ ), which results into a total recording time of $19 \mathrm{~min}$ for scanning from 1322.5 to $1226.5 \mathrm{~cm}^{-1}$. All spectra were treated with a high pass Fourier filter (Gaussian apodization) in MATLAB (MathWorks, Natick, MA, USA) blocking all contributions with a bandwidth of $\geq 100 \mathrm{~cm}^{-1}$ to correct for time-dependent baseline drifts and were Fourier-smoothed for a final resolution of $5 \mathrm{~cm}^{-1}$. 


\section{FT-IR and FT-VCD measurements}

FT-VCD and FT-IR spectra of $200 \mathrm{mM}$ solutions of $\mathbf{1}$ and $\mathbf{2}$ in $\mathrm{CDCl}_{3}$ were recorded on a Bruker Tensor 27 FTIR spectrometer equipped with a Bruker PMA 50 VCD side-bench module with a resolution of $4 \mathrm{~cm}^{-1}$ in a $110 \mu \mathrm{maF}_{2}$ cell. The spectra represent the average of 5 hrs of measuring followed by solvent subtraction. For VCD measurements between 1800 and $800 \mathrm{~cm}^{-1}$ the photoelastic modulator (PEM) was set to quarter-wave retardation at 1400 $\mathrm{cm}^{-1}$.

\section{Singular value decomposition and global fitting}

Background information on the applications of singular value decomposition to the analysis of spectroscopic data can be found elsewhere. ${ }^{17}$ Briefly, the data matrix $\mathbf{A}(361 \times 20)$, containing baseline-corrected spectra as columns and time-traces as rows, was factorized with SVD as: $\mathbf{A}=\mathbf{U} \times \mathbf{S} \times \mathbf{V}^{\mathbf{T}}$. The matrices $\mathbf{U}(361 \times 20)$ and $\mathbf{V}(20 \times 20)$ contain as columns orthonormal "component" spectra (abstract spectra) and time-traces of decreasing relevance. The contribution of each spectro-temporal component to describe the experimental matrix $\mathbf{A}$ by means of least squares is given by its singular value contained in the diagonal of the $\mathbf{S}$ $(20 \times 20)$ matrix. SVD was performed in MATLAB.

Global exponential fitting was performed in MATLAB by implementing the LevenbergMarquardt algorithm ${ }^{18}$ in home-made routines, as described previously. ${ }^{19}$ The columns of the matrix $\mathbf{V}$, obtained from SVD of the experimental matrix, were analyzed following standard procedures for combining SVD and fitting, as described in the literature. ${ }^{20}$ The nonlinear part of global exponential fitting, the estimation of the time constant(s), was performed on a reduced set of columns of $\mathbf{V}$. Because the spectrum at $t_{0}$ was a zero line, constraints were set to force the fit to go through zero. The estimation of the exponential amplitudes versus the wavenumber, known as decay associated spectra (DAS), made use of all the columns of $\mathbf{V}$. For more accurate results we weighted the data in respect to wavenumbers for both SVD and global exponential fitting, based on wavenumber-dependent standard deviations obtained from measuring a $\mathrm{CHCl}_{3}$ background spectrum.

\section{Results}

The time-scale of the hydrolysis of $\mathbf{1}$ can be controlled by different parameters. High pH, i. e. a high concentration of hydroxide ions, provides a large excess of precipitating agent and 
hence shifts the equilibrium to the reaction side, thereby releasing free base 2. Our reaction setup was designed as a two-phase system consisting of an aqueous sodium hydroxide solution and complex 1 dissolved in chloroform $\left(\mathrm{CHCl}_{3}\right)$. Therefore, the kinetics depended on the area of the organic/water interface, where the reaction took place. The course of the reaction could be visually inspected from decoloration of the chloroform phase and formation of green precipitate of $\mathrm{Ni}(\mathrm{OH})_{2}$ at the interface (Fig. 1).

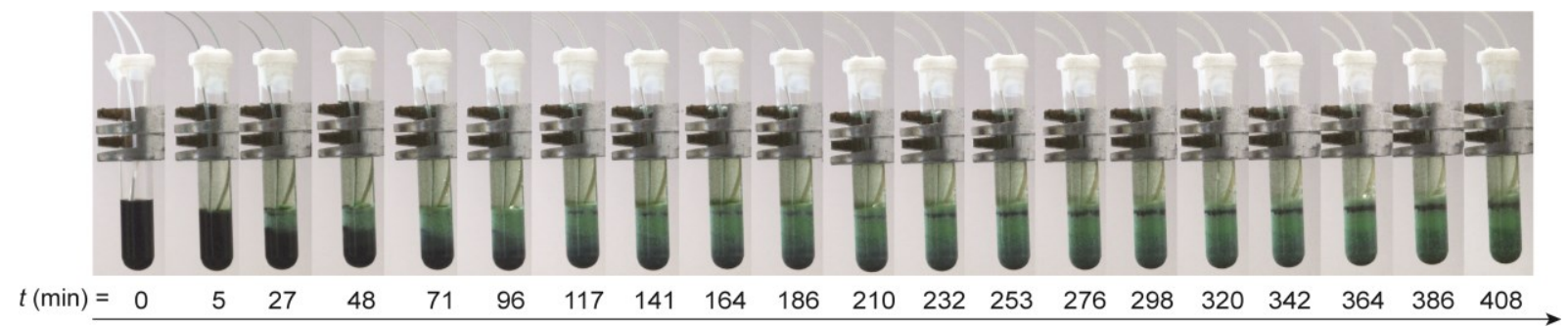

Fig. 1: Photographs of the reaction vessel taken at the indicated times.

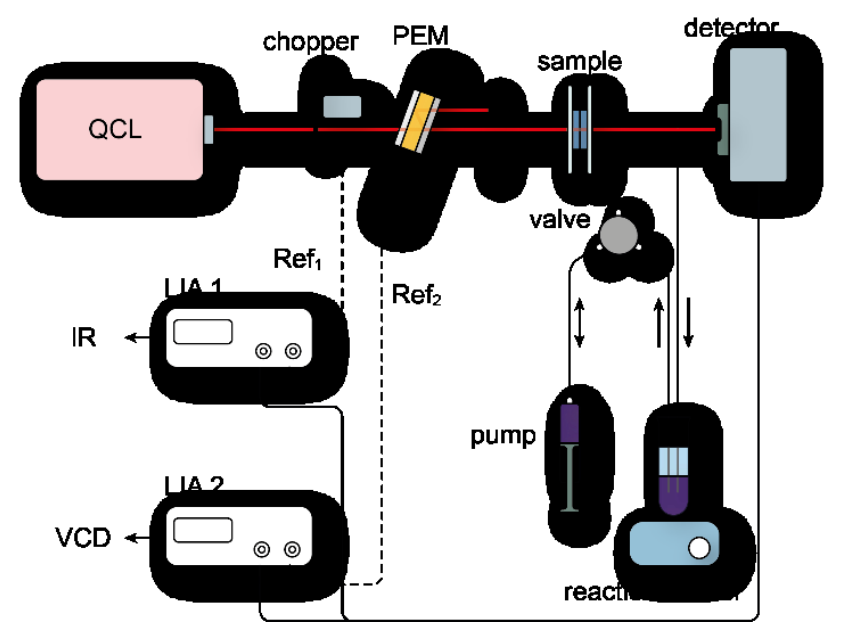

Fig. 2: Experimental setup. QCL: external cavity quantum cascade laser; PEM: photoelastic modulator; LIA: lock-in amplifier; $\operatorname{Ref}_{1}$ : reference frequency of the chopper; $\operatorname{Ref}_{2}$ : reference frequency of the PEM.

The sample solution was aspirated from the chloroform phase and pumped through a $200 \mu \mathrm{m}$ $\mathrm{CaF}_{2}$ cell between two consecutive measurements. After a spectrum was recorded, the solution was re-injected into the reaction vessel. Because the reaction only takes place at the interface, every sample taken from the chloroform phase is a snapshot of the composition of the reaction solution at a certain point in time. For recording time-dependent IR absorbance and VCD spectra in the spectral range from 1322.5 to $1226.5 \mathrm{~cm}^{-1}$ we used an instrumental setup similar to the one that had been introduced previously (Fig. 2). ${ }^{7}$ The high throughput of IR light from the QCL was sufficient to overcome the background from $\mathrm{CHCl}_{3}$, which has an optical density (OD) between 1 and 2 in the spectral range from 1238 to $1230 \mathrm{~cm}^{-1}$. As a consequence of intermittent instead of continuous pumping, the reaction proceeded for the 
portion that remained in the reaction vessel but was halted for the portion that rested in the sample cell and the tubing during each measurement (19 min). This was necessary to ensure that the intensity at every wavenumber in the IR and VCD spectra corresponded to the same snapshot within the course of reaction. Mixing back the sample from the cell to the reaction batch instead of discarding it, however, lead to observation of an artificially retarded time constant $\tau_{\text {eff }}$ instead of the true time constant $\tau$, which actually reflects the reaction rate. The difference between $\tau_{\text {eff }}$ and $\tau$ gets negligible for large reaction batches or can be minimized by increasing the time resolution of the measurement, for example by scanning a smaller spectral range.

For background correction of the IR and VCD spectra, we subtracted the spectrum recorded before the sodium hydroxide solution layer was added (spectrum at $t_{0}$ ). The IR and VCD difference spectra recorded over 408 min are shown in Fig. 3 A. 

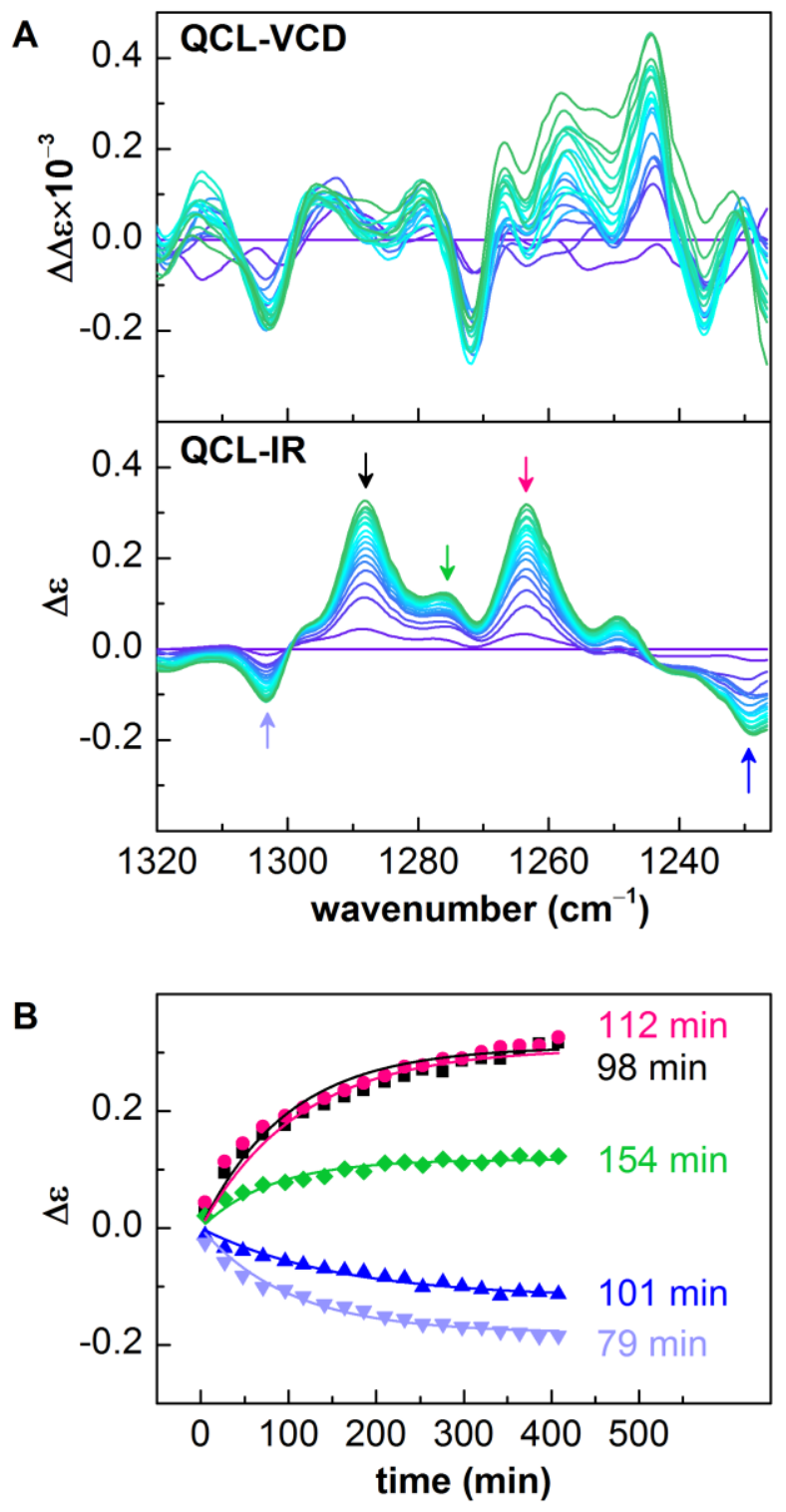

Fig. 3: A: Time-dependent QCL VCD and IR spectra. Because the spectrum at $t_{0}$ was used as a background, the difference absorbance for $t_{0}$ is a zero line. Color coding is from purple $\left(t_{0}\right)$ to lime-green $\left(t_{408} \min \right)$, according to the time-dependent color change of the reaction solution. Spectra are corrected for baseline drifts and smoothed as described in the Materials and Methods section. B: Time traces for IR difference absorbance at individual wavenumbers (indicated by arrows in A). The times are the effective time constants from exponential fitting of the IR absorbance change at 1303 (light blue), 1288 (black), 1276 (green), 1264 (pink), and 1228 (blue) $\mathrm{cm}^{-1}$.

The spectrum recorded at $t_{0}$ corresponds to pure $\mathbf{1}$. By subtracting this spectrum from eq. 1 and substituting $\tau$ by $\tau_{\text {eff }}$ we obtain

$$
\Delta A(\widetilde{v}, t)=a(\widetilde{v})\left(e^{-\frac{t}{\tau_{e f}}}-1\right) .
$$


This means that at $t_{0}$ the time-dependent data set starts with a zero line and ends after total conversion $\left(t_{\infty}\right)$ with the full negative amplitude spectrum $(-a)$, corresponding to 2 minus $\mathbf{1}$. We tested this model by fitting eq. 2 to the time-dependent change in IR absorbance at different wavenumbers corresponding to characteristic maxima or minima in the IR difference spectrum (Fig. 3 A). Fits at 1303, 1288, 1276, 1264, and $1228 \mathrm{~cm}^{-1}$ delivered a time constant of $109 \mathrm{~min}$ in average, which is reasonable for the time frame of this reaction, though with values varying in the range between 79 and $154 \mathrm{~min}$ (Fig. 3 B). The variations arise from fitting two parameters the same time: the amplitude of absorbance and the time constant. Therefore, inaccuracies in the amplitude also result in errors for the determination of the time constant. This is even more evident for VCD, where absorbance values at single wavelengths are more error-prone, because the amplitude of a VCD spectrum is regularly $\sim 10^{-4}$ of the corresponding IR signal. ${ }^{21}$ Particularly for the evaluation of three-dimensional VCD data, fitting at multiple instead of individual wavenumbers is much more accurate and less biased. Furthermore, given that the model is correct, global exponential fitting of three-dimensional spectral data provides spectral information about the decay associated components involved in the process. Such decay associated VCD spectra can provide information about the presence or absence of a particular substance, as well as about its configuration and its conformation.

Therefore, global exponential fitting of eq. 2 to the data from Fig. 3 should both deliver the amplitude spectrum $a$, and the time constant $\tau_{\text {eff }}$ for the exponential decay of $a$ in the experimental data. Prior to global exponential fitting, we performed singular value decomposition (SVD) of the time-dependent data set (Fig. 4). As expected, only the first abstract spectrum (Fig. 4 A, I) showed an exponential time-dependence (Fig. 4 B, I). The second extracted component mostly represents a linear time-drift (Fig. 4 B, II) of the baseline (Fig. 4 A, II). All other components can mainly be attributed to noise, based on their random time-evolution (see Fig. 4 B, III and IV). 

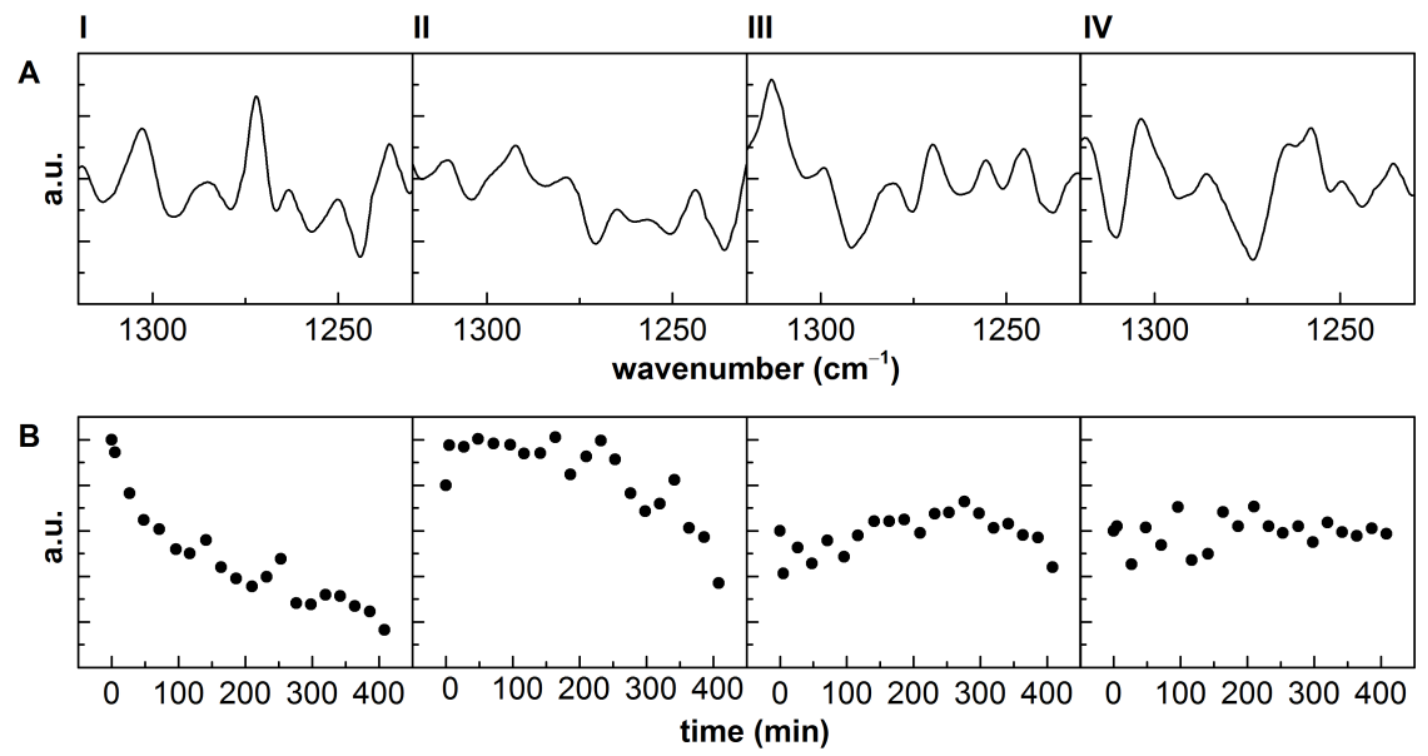

Fig. 4: Spectra (A) and time traces of the corresponding coefficients (B) of the first four components from SVD analysis.

Instead of performing a global exponential fit on the full data set, it is more efficient to perform the fit on the time-dependent information in $\mathbf{V}$, which delivers $\tau_{\text {eff, and additionally }}$ allows for calculating back the pure amplitude spectrum $a$ (see Materials and Methods section for details). The results are shown in Fig. 5. The positive and negative VCD difference bands in the amplitude spectrum $a$ (Fig. $5 \mathrm{~B}$ ) represent the $\mathrm{C}-\mathrm{H}$ bending modes of $2{ }^{22}$ which appear as enhanced VCD signals in the spectrum of $\mathbf{1}$. The spectrum also agrees with a difference VCD spectrum of 1 minus 2 measured on a FT-VCD instrument (Bruker PMA 50) using $\mathrm{CDCl}_{3}$ as a solvent instead of $\mathrm{CHCl}_{3}$ (Fig. 5 A). The coefficients for $a$ plotted against time (Fig. 5 C) clearly show slow exponential behavior with $\tau_{\text {eff }}=127 \mathrm{~min}$. This is a similar result to that achieved by fitting the absorbance change at individual wavenumbers in the IR (see above). The residuals (experimental minus fitted spectra), exemplarily calculated for $t=48$, 186, and $342 \mathrm{~min}$ (Fig. 5 D), contain noise and background artifacts. We also performed global exponential fitting on the time-dependent QCL-IR spectra (supporting information) leading to $\tau_{\text {eff }}=123 \mathrm{~min}$. This control analysis was necessary to demonstrate the reliability of VCD-based kinetic studies, which is particularly important for those cases in which characteristic time-dependent changes are observed in the VCD, but not in the IR. 

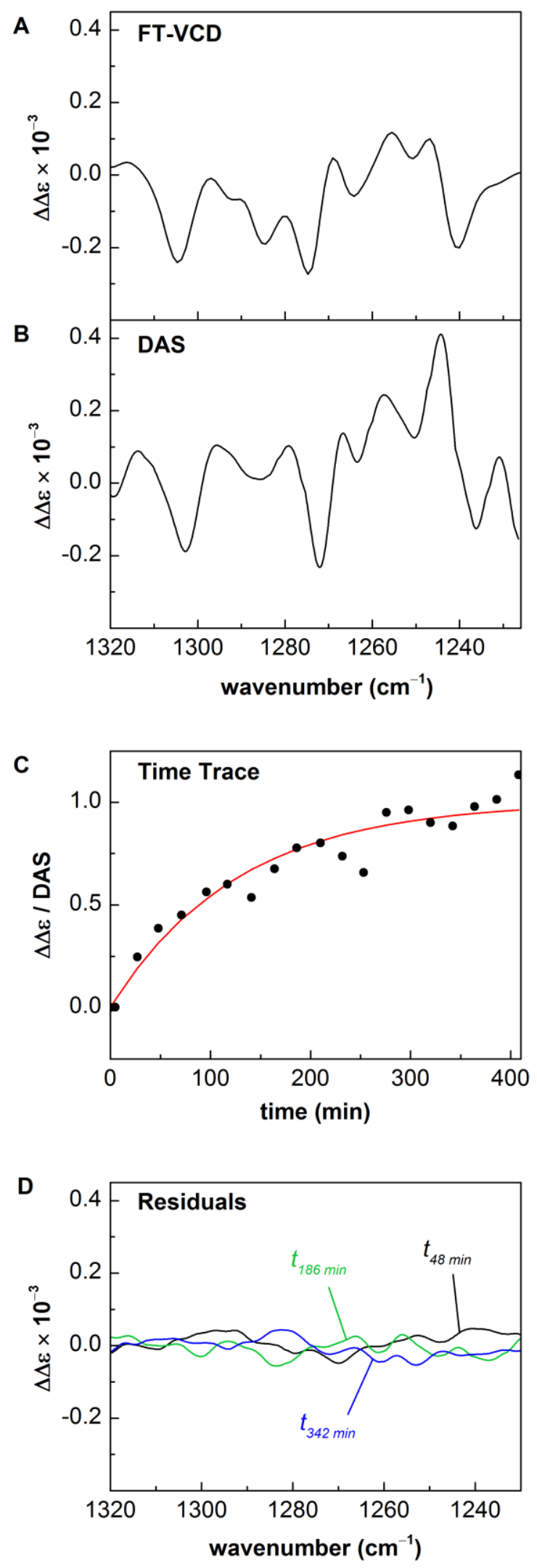

Fig. 5 A: Difference spectrum of pure complex 1 minus free base $2\left(200 \mathrm{mM}\right.$ in $\left.\mathrm{CDCl}_{3}\right)$ measured on a FT-VCD spectrometer. B: Decay associated spectrum (DAS) from global exponential fitting of time-dependent QCL- 
VCD data of the hydrolysis reaction in $\mathrm{CHCl}_{3}$. This spectrum corresponds to the difference of $\mathbf{1}$ minus 2 . C: Back calculated fractions of decay associated spectrum $a$ versus time with the theoretical curve using eq. 2 and $\tau_{\text {eff }}=127$ min. D: Exemplary residuals (experiment minus fit) for the global exponential fit at $t=48,186$, and $342 \mathrm{~min}$.

A pure VCD spectrum of reaction product 2 was obtained from subtracting the decay associated spectrum (1 minus 2, Fig. $5 \mathrm{~B}$ ) from the $\mathrm{CHCl}_{3}$-background corrected spectrum recorded at $t_{0}$, representing 1. In Fig. 6 these spectra of $\mathbf{1}$ and $\mathbf{2}$ are compared to the corresponding spectra recorded on a FT-VCD spectrometer in $\mathrm{CDCl}_{3}$. All characteristic features in the FT-VCD (Fig. $6 \mathrm{~A}$ ) are also present in the spectra from the QCL-VCD experiment (Fig. 6 B). Additional bands, mainly visible in the case of 2 (Fig. 6 B II), are due to background artifacts. However, by using $t_{0}$ as a background, as performed in this study, most of these artifacts seem to cancel out. Global exponential fitting of double-difference time-dependent VCD spectra lead to identification of product $\mathbf{2}$ from the spectrum associated to an exponential decay with a time constant of $127 \mathrm{~min}$. This supports a pseudo-first order kinetic model for the hydrolysis of $\mathbf{1}$, and, moreover, demonstrates the applicability of QCLVCD for monitoring a chemical reaction.
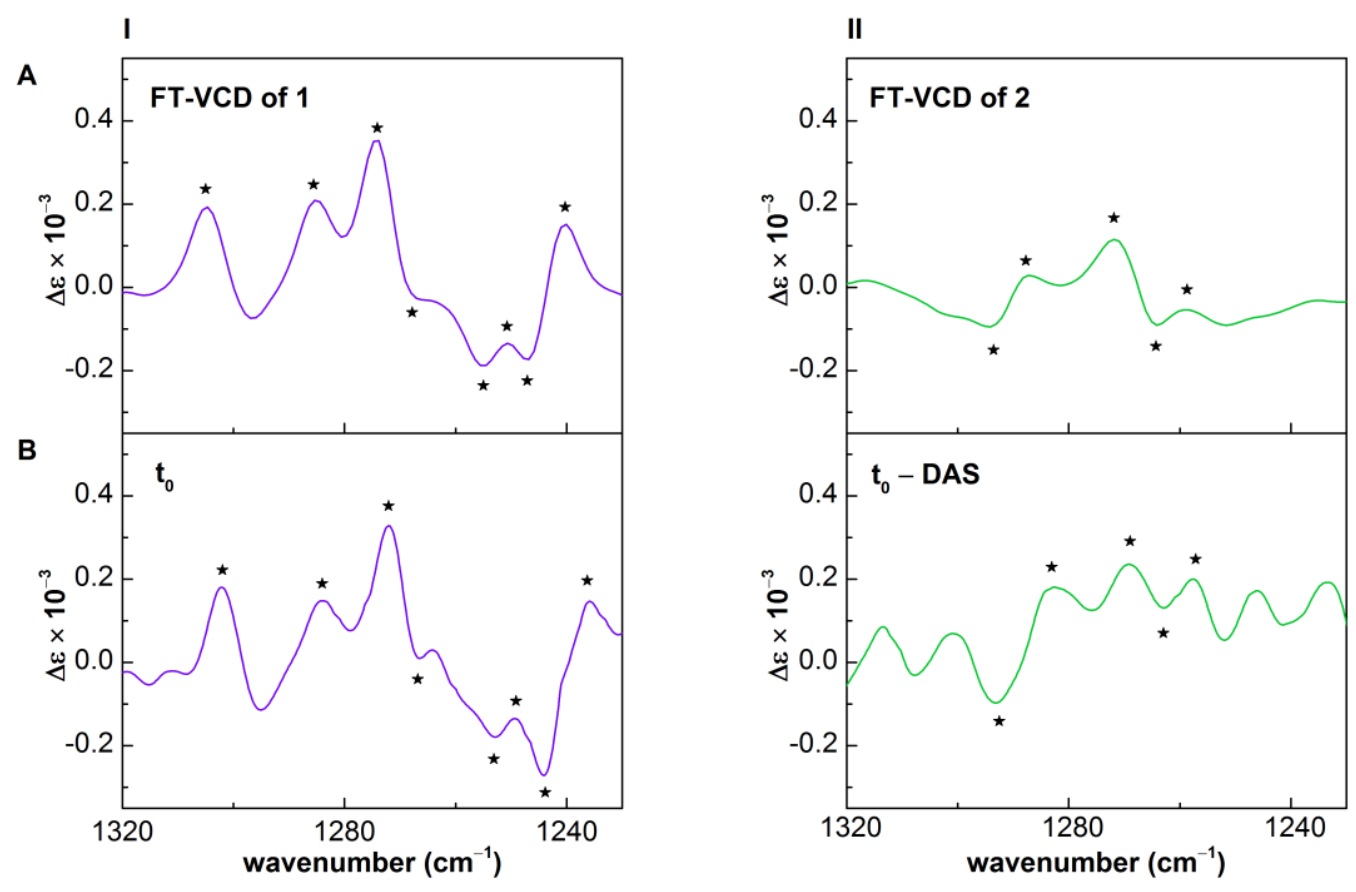

Fig. 6 A: FT-VCD spectra of $200 \mathrm{mM} \mathrm{CDCl}_{3}$ solutions of 1 (I) and 2 (II). B: Spectrum recorded at $t_{0}$ of the QCLVCD reaction monitoring experiment (corrected for $\mathrm{CHCl}_{3}$-background) representing pure 1 (I) and spectrum of 
pure 2 constructed by subtracting DAS from the spectrum at $t_{0}$. Characteristic spectral features are indicated by asterisks.

\section{Discussion}

The hydrolysis of complex $\mathbf{1}$ to free base $\mathbf{2}$ is an excellent model for a reaction comprising significant changes in the VCD that can be monitored over time. The observed spectral changes agree with the expected difference of the individual VCD spectra for $\mathbf{1}$ and $\mathbf{2}$, respectively, and occur with a similar time constant as obtained from exponential fitting of IR absorbance at individual wavenumbers. VCD reaction monitoring is generally challenging due to strong background absorbance of solvents that are usually applied for chemical reactions. The use of a tunable QCL as a bright light source allows for measuring samples with an OD of up to 3.5. ${ }^{7}$ Dedicated solvents for vibrational spectroscopy, such as deuterated chloroform $\left(\mathrm{CDCl}_{3}\right)$ show low absorbance over a broad spectral range in the mid IR. At a path length of $200 \mu \mathrm{m} \mathrm{CDCl}_{3}$ has a maximal $\mathrm{OD}$ of 0.03 in the spectral range from 1322.5 to $1226.5 \mathrm{~cm}^{-1}$. For the same path length, the $\mathrm{OD}$ of $\mathrm{CHCl}_{3}$ is 0.06 at $1320 \mathrm{~cm}^{-1}$, but, due to absorbance of the $\mathrm{C}-\mathrm{H}$ bending vibration, it increases to 1.0 at 1238 and exceeds 2 below $1230 \mathrm{~cm}^{-1}$, which is still feasible with the use of the QCL light source.

Analogous to dispersive instruments, in QCL-VCD a wider range requires a longer acquisition time, which increases even more with smaller scanning steps for a higher resolution. Because the signals are detected individually at every single wavenumber, the spectral range and regions with higher resolution can be restricted to signals and motifs that are necessary for an unambiguous analysis. Thereby, sampling of redundant spectral regions, which are nontransparent even for the QCL, can be avoided. This is a major difference to Fourier transform spectroscopies, where all wavelengths are sampled simultaneously and spectra are principally obtained with uniform resolution. For monitoring changes in optical activity in a broad range of different reactions, however, it might be advantageous to optimize for a particular spectral region of interest that is detectable with a particular solvent background. Optimization of the sampled spectral region might also lead to increased timeresolution, which is for QCL-VCD still limited by an integration time of milliseconds to seconds per wavenumber for a reasonable signal to noise ratio, and a certain stabilization time required by the laser after stepping from one wavenumber to another. Recently, setups for laser-based pump-probe VCD measurements have been introduced that technically allow for picosecond $^{23}$ or femtosecond ${ }^{24}$ time-resolution. Synthetic reactions, however, often happen on the time-scale of minutes to hours and usually do not fulfill the requirements for pump-probe 
experiments. $^{25}$ Data acquisition does not necessarily have to be very fast in monitoring synthetic reactions. Because averaging of many transient spectra is difficult to accomplish, duration of data acquisition has to be optimized in respect to signal-to-noise on one hand and to time-resolution on the other hand. In this study, we monitored spectral changes $(\Delta \Delta \varepsilon \sim 6 \times$ $10^{-4} \mathrm{~L} \mathrm{~mol}^{-1} \mathrm{~cm}^{-1}$ ), which were, due to electronic enhancement, about 10 fold larger than signal changes that would have been expected for a reaction involving simple organic molecules $\left(\Delta \Delta \varepsilon \sim 10^{-5}-10^{-4} \mathrm{~L} \mathrm{~mol}^{-1} \mathrm{~cm}^{-1}\right)$. Measuring these subtle changes will require improvements to the experimental setup to increase baseline-stability and signal-to-noise. However, if an appropriate least-squares regression is applied on the whole data set, information about the time-dependent process can even be drawn out of moderate signal-tonoise data.

\section{Conclusions}

In this study, we demonstrate the monitoring of a chemical reaction by observing timedependent changes of VCD signals over time. We recorded time-dependent VCD spectra with a QCL-based setup. The strong light source extends the applicability of VCD reaction monitoring to strongly absorbing samples, which is the case for any solution with a solvent that exhibits infrared absorption in the observed spectral range. As shown for time-dependent VCD spectra of the hydrolysis of 1 into 2, a combination of SVD and global fitting was used to support an assumed reaction model, followed by the identification of reactant and product spectra, and the determination of kinetic parameters for the reaction. This could in particular be valuable for applications in stereoselective syntheses. VCD reaction monitoring could help to optimize reaction conditions, e. g., in syntheses where optical purity reaches an optimum but decreases before full conversion of a substrate to a chiral product is achieved. ${ }^{26}$ Here, VCD only monitors the fate of the substance of interest, a chiral molecule, ignoring all the background from achiral solvent and reactants.

\section{Acknowledgments}

The authors thank Dr. A. Lambrecht for the loan of the QCL, and Prof. M. Müller and Prof. P. Fischer for helpful discussions. We acknowledge the use of the computing resources provided by the Black Forest Grid Initiative. S. L. is indebted to the Baden-Württemberg Stiftung for the financial support of this research project by the Eliteprogramme for Postdocs. S. L. also thanks the Eugen-Graetz-Stiftung for financial support. 


\section{Literature cited}

1. Lewis JW, Tilton RF, Einterz CM, Milder SJ, Kuntz ID, Kliger DS. New technique for measuring circular dichroism changes on a nanosecond time scale. Application to (carbonmonoxy)myoglobin and (carbonmonoxy)hemoglobin. J Phys Chem 1985;89:289-294.

2. Hache F. Application of time-resolved circular dichroism to the study of conformational changes in photochemical and photobiological processes. J Photochem Photobiol, A 2009;204:137-143.

3. Mendonça L, Hache F. Nanosecond $T$-jump experiment in poly(glutamic acid): a circular dichroism study. Int J Mol Sci 2012;13:2239-2248.

4. Freedman TB, Cao XL, Dukor RK, Nafie LA. Absolute configuration determination of chiral molecules in the solution state using vibrational circular dichroism. Chirality 2003;15:743-758.

5. Izumi H, Ogata A, Nafie LA, Dukor RK. A revised conformational code for the exhaustive analysis of conformers with one-to-one correspondence between conformation and code: application to the VCD analysis of $(S)$-ibuprofen. J Org Chem 2009;74:1231-1236.

6. Keiderling TA. Conformational studies of biopolymers, peptides, proteins, and nucleic acids. A role for vibrational circular dichroism. In: Berova N, Polavarapu PL, Nakanishi K, Woody RW, editors. Comprehensive chiroptical spectroscopy. Volume 2. Hoboken, New Jersey: John Wiley \& Sons, Inc.; 2012. pp 707-758.

7. Lüdeke S, Pfeifer M, Fischer P. Quantum-cascade laser-based vibrational circular dichroism. J Am Chem Soc 2011;133:5704-5707.

8. Guo CN, Shah RD, Dukor RK, Cao XL, Freedman TB, Nafie LA. Determination of enantiomeric excess in samples of chiral molecules using fourier transform vibrational circular dichroism spectroscopy: simulation of real-time reaction monitoring. Anal Chem 2004;76:6956-6966.

9. Guo CN, Shah RD, Mills J, Dukor RK, Cao XL, Freedman TB, Nafie LA. Fourier transform near-infrared vibrational circular dichroism used for on-line monitoring the epimerization of 2,2-dimethyl-1,3-dioxolane-4-methanol: A pseudo racemization reaction. Chirality 2006;18:775-782.

10. Hsu C-PS. Infrared spectroscopy. In: Settle F, editor. Handbook of instrumental techniques for analytical chemistry. Upper Saddle River, New Jersey: Prentice-Hall; 1997. pp 247-283.

11. Müller KH, Plesser T. Variance reduction by simultaneous multiexponential analysis of data sets from different experiments. Eur Biophys J 1991;19:231-240.

12. Freedman TB, Nafie LA. Vibronic coupling calculations of vibrational circulardichroism intensities using floating basis-sets. J Chem Phys 1988;89:374-384.

13. He YN, Cao XL, Nafie LA, Freedman TB. Ab initio VCD calculation of a transitionmetal containing molecule and a new intensity enhancement mechanism for VCD. J Am Chem Soc 2001;123:11320-11321.

14. Nafie LA. Theory of vibrational circular dichroism and infrared absorption: extension to molecules with low-lying excited electronic states. J Phys Chem A 2004;108:72227231.

15. Mattigod SV, Rai D, Felmy AR, Rao LF. Solubility and solubility product of crystalline $\mathrm{Ni}(\mathrm{OH})_{2}$. J Solution Chem 1997;26:391-403.

16. Mason SF, Peacock RD. Complexes of some first-row transition-elements with (-)spartein. J Chem Soc, Dalton Trans 1973:226-228.

17. Hendler RW, Shrager RI. Deconvolutions based on singular-value decomposition and the pseudoinverse: a guide for beginners. J Biochem Bioph Meth 1994;28:1-33. 
18. Press WH. Numerical recipes: the art of scientific computing. Cambridge: Cambridge University Press; 2007.

19. Lórenz-Fonfría VA, Kandori H. Bayesian maximum entropy (two-dimensional) lifetime distribution reconstruction from time-resolved spectroscopic data. Appl Spectrosc 2007;61:428-443.

20. Hendler RW, Bose SK, Shrager RI. Multiwavelength analysis of the kinetics of reduction of cytochrome $a a_{3}$ by cytochrome $c$. Biophys J 1993;65:1307-1317.

21. Nafie LA. Vibrational optical activity. Principles and applications. Chichester: John Wiley \& Sons; 2011.

22. Bouř P, McCann J, Wieser H. Vibrational circular dichroism study of (-)-sparteine. J Phys Chem A 1997;101:9783-9790.

23. Bonmarin M, Helbing J. A picosecond time-resolved vibrational circular dichroism spectrometer. Opt Lett 2008;33:2086-2088.

24. Rhee HJ, June YG, Lee JS, Lee KK, Ha JH, Kim ZH, Jeon SJ, Cho MH. Femtosecond characterization of vibrational optical activity of chiral molecules. Nature 2009;458:310-313.

25. Zewail AH. Femtochemistry. Past, present, and future. Pure Appl Chem 2000;72:2219-2231.

26. Chen CS, Fujimoto Y, Girdaukas G, Sih CJ. Quantitative analyses of biochemical kinetic resolutions of enantiomers. J Am Chem Soc 1982;104:7294-7299. 